\title{
Tactile short-term memory for stimuli presented on the fingertips and across the rest of the body surface
}

\author{
Malika Auvray • Alberto Gallace • Charles Spence
}

Published online: 19 February 2011

(C) Psychonomic Society, Inc. 2011

\begin{abstract}
The aim of this study was to investigate the extent to which tactile information that is unavailable for full conscious report can be accessed using partial-report procedures. In Experiment 1, participants reported the total number of tactile stimuli (up to six) presented simultaneously to their fingertips (numerosity judgment task). In another condition, after being presented with the tactile display, they had to detect whether or not the position indicated by a (visual or tactile) probe had previously contained a tactile stimulus (partial-report task). Participants correctly reported up to three stimuli in the numerosity judgment task, but their performance was far better in the partial-report task: Up to six stimuli were perceived at the shortest target-probe intervals. A similar pattern of results was observed when the participants performed a concurrent articulatory suppression task (Exp. 2 ). The results of a final experiment revealed that performance in the partial-report task was overall better for stimuli presented on the fingertips than for stimuli presented across the rest of the body surface. These results demonstrate that tactile information that is unavailable for
\end{abstract}

M. Auvray $(\square)$

Laboratoire d'Informatique pour la Mécanique et les Sciences de l'Ingénieur (LIMSI), CNRS UPR 3251,

B.P. 133, 91403 Orsay Cedex, France

e-mail:malika@malika-auvray.com

\section{A. Gallace}

Dipartimento di Psicologia,

Universita' degli Studi di Milano Bicocca,

Milano, Italy

M. Auvray · A. Gallace $\cdot$ C. Spence

Crossmodal Research Laboratory,

Department of Experimental Psychology, Oxford University,

Oxford, England report in a numerosity task can nevertheless sometimes still be accessed when a partial-report procedure is used instead.

Keywords Touch · Short-term memory · Iconic memory · Numerosity Partial report $\cdot$ Spatial representation

Although our sensory experience seems to allow conscious access to detailed information, many important studies of short-term memory (STM) have revealed that observers only have access to a relatively small percentage of the information available in a scene at any one time. For instance, research on visual STM has shown that observers are only able to remember the identity of about 4 or 5 letters out of 12 or more, even if they have the impression that they can see all of them. It thus appears that the storage of information in visual STM is very limited (see, e.g., Coltheart, 1983).

The question that naturally arises when such limitations in STM are considered concerns the number of objects we are aware of at any one time, which James (1890/1950) termed the "span of consciousness" (e.g., Fernberger, 1921; Kaufman, Lord, Reese, \& Volkmann, 1949; see Gallace \& Spence, 2008, for a review). In particular, numerosity judgments provide a means of investigating the span of consciousness for (and between) the different sensory modalities. This technique typically consists of asking observers to count the number of stimuli that are presented simultaneously. In vision, studies of numerosity judgments have revealed that people are able to count a small number of items (i.e., between one and four stimuli) rapidly and near perfectly. Above this number, the percentage of errors that participants make increases, as do their reaction times (see Trick \& Pylyshyn, 1994, for a review). In addition, it should be noted that this increase is such that it gives rise to a discontinuity in 
the slopes of the error and reaction time functions, which has led many authors to distinguish between two different enumeration processes: "subitizing," defined as fast and accurate, and "counting," defined as slow and error prone (e.g., Mandler \& Shebo, 1982; Peterson \& Simon, 2000). More specifically, subitizing in vision has been estimated to occur for up to (i.e., including) three (see, e.g., Chi \& Klahr, 1975) or four (e.g., Atkinson, Campbell, \& Francis, 1976; Jevons, 1871; Simons \& Langheinrich, 1982; Trick \& Pylyshyn, 1994) stimuli. In audition, Kashino and Hirahara (1996) used a simultaneous stimulus presentation procedure to show that participants can estimate almost perfectly the number of talkers for up to two different voices, with their performance decreasing with additional talkers (see also Camos \& Tillmann, 2008, for evidence from an auditory sequential-presentation task).

In the tactile modality, the percentage of errors in counting the number of body locations stimulated at any one time becomes very high (i.e., error rates exceed 30\%) when three vibrotactile stimuli are presented, and most of the time performance falls to chance level when four or more stimuli are presented (see Gallace, Tan, \& Spence, 2006; see also Riggs et al., 2006, for stimuli presented on the fingertips). Note that Gallace and his colleagues (Gallace, Tan, Haggard, \& Spence, 2008; Gallace et al., 2006) found that the accuracy of participants' responses decreased linearly with an increase in the number of stimuli, which suggested no clear distinction between counting and subitizing in touch (although note that Riggs et al., 2006, did find a distinction between the two enumeration processes in touch; see Gallace, Tan, \& Spence, 2008, for a fuller discussion of this controversy). It is worth noting here that a study by Gallace, Tan, and Spence (2007) investigated numerosity judgments for simultaneously presented visual and tactile stimuli. The results revealed that multisensory numerosity judgments were significantly less accurate than would be predicted by an account that assumes independent, modality-specific resources. In other words, in Gallace et al.'s (2007) study, numerosity judgments for stimuli presented simultaneously in vision and touch were less accurate than would be predicted if one were to sum the performance obtained when the stimuli were presented independently to each modality.

It should be noted that the question of whether there is a single capacity limit or different capacity limits is controversial. According to Wickens (1984), there is not a single capacity limit (or pool of resources), but rather separate limits (or pools of resources) for separate abilities (e.g., visual and auditory or spatial and verbal; see also Hancock, Oron-Gilad, \& Szalma, 2007, for an extension of the multiple-resources approach to incorporate the tactile modality). On the other hand, according to Cowan (2001), there does appear to be a single, central capacity limit that can be identified with the focus of attention. Such a pure capacity limit, which is the same regardless of the stimulus modality involved, averages about four items. According to Cowan (2001), a distinction has to be made between pure and compound STM capacity limits. Pure STM capacity limits can be observed when rehearsal and long-term memory cannot be used to combine stimulus items into larger units, and when storage mechanisms that are not capacity limited (such as sensory, phonological, or spatial stores) do not allow the capacity-limited storage mechanism to be refilled during recall. On the other hand, when these conditions are not fulfilled - that is, when the number of separately held items is unclear - the compound STM limits (which can involve more items than the pure STM limits) can be observed.

An important issue regards the fate of information that has not been explicitly reported (e.g., in numerosity judgment tasks): Was the information that was not reported nevertheless stored, without conscious access to it? Indeed, it has been shown for the various senses that information that has not been explicitly reported during full-report procedures can still be accessed when partial-report procedures are used instead. For instance, Sperling (1960) demonstrated that participants can report an average of four or five letters when they are presented for $500 \mathrm{~ms}$ in visual matrices. However, when a partial-report procedure has been used instead, which consisted of an auditory probe indicating the row of the matrix that had to be reported, the storage capacity of participants was estimated at 12-16 visual items. Sperling (1960) interpreted the difference between the two procedures as being attributable to the rapid temporal decay of the visual information, which prevented the recall of all of the items during the full-report task. These items were nevertheless stored in a shorterlasting visual representation, which has been termed "iconic memory" (see Coltheart, 1983; Neisser, 1967, for reviews). An equivalent, very short-term form of memory has also been reported in the auditory modality, where it has been labeled "echoic memory" (e.g., Cowan, 1984). In particular, in a study by Darwin and colleagues (Darwin, Turvey, \& Crowder, 1972; see also Treisman \& Roston, 1972), participants had to report the stimuli presented from three different spatial locations (left ear, middle of the head, and right ear). The performance of participants was lower when they were asked to report all of the stimuli than when they were asked, via a visual cue, to report only the stimuli coming from one of the three locations. In addition, performance was only better in the partial-report task, as compared to the whole-report procedure, when the cue was presented shortly after the stimuli.

Finally, a short-term form of storage has been reported in the tactile modality as well. In an early study, Bliss and 
colleagues (Bliss, Crane, Mansfield, \& Townsend, 1966; see also Hill \& Bliss, 1968; see Gallace \& Spence, 2009, for a recent review) had their participants associate the 24 interjoint segments of the fingers of both hands (excluding the thumbs) with a letter of the alphabet. Next, up to 12 stimuli were presented to participants on these interjoint segments, and the participants had to try to report all of the positions that were stimulated using the corresponding alphabetical labels. Using this technique, the participants were able to correctly report an average of 3.5 positions. However, when Bliss et al. used a partial-report procedure consisting of cuing one of the locations (i.e., the 8 topmost interjoint positions, the 8 in the middle, or the 8 situated at the bottom of the fingers) instead and asked participants to determine whether or not this location had been stimulated previously, the average number of correct responses increased to 4.5 positions (i.e., 1 more position than when the full procedure was used).

The results of Bliss et al.'s (1966) study might therefore be taken to suggest the existence of a tactile STM store, equivalent to the iconic memory found in vision. However, the poor performance of participants in the full-report task might also reflect the difficulty of the response method that was used (i.e., associating each of the interjoint segments with a letter of the alphabet), which may have diminished the participants' memory capacities. Following on from this hypothesis, Gallace et al. (2008) used a simpler "fullreport" procedure task - namely, a numerosity judgment task. In this study, the participants were presented with displays consisting of up to seven vibrotactile stimuli on their body surface. Whereas the participants reported up to three stimuli correctly in the numerosity judgment task, their performance increased to the estimated report of five stimuli during a partial-report task, which involved asking the participants whether or not a specific body part had received tactile vibrations, using a tactile probe.

Taken together, these studies reveal that there is a tactile form of STM equivalent to the iconic memory reported in vision. However, three important questions still remain to be answered. First, is STM unimodal in nature (i.e., does it belong strictly to the tactile register), or is it instead multisensory/amodal in nature? Indeed, given the existence of extensive crossmodal links in spatial attention and in the cognitive and neural systems underlying the representation of space (see, e.g., Spence \& Driver, 2004, for a review), one might predict that the processes underlying the encoding of tactile stimuli presented on the body have multisensory components as well. If this were to be the case, the modality of presentation of the probe should not have any effect on participants' performance. In order to investigate this specific question, Experiment 1 was designed to incorporate a partial-report task with both tactile and visual probes.
Second, given that the participants' ability to report tactile positions was not affected by the nature (i.e., the modality) of the probe in Experiment 1, the question arose as to whether or not the encoding of these positions was semantic. In order to investigate this question, Experiment 2 involved an articulatory suppression task that allowed us to control for any semantic encoding of the positions of the tactile stimuli.

The final experiment reported here investigated whether or not similar processes underlie the processing of tactile stimuli presented on the fingertips and across the rest of the body surface. It is certainly true that people more frequently attend to tactile stimuli presented to their fingers than to those presented elsewhere on their body; in addition, relative to the surface area concerned, more neural processing is given over to the hands than to other parts of the body surface (see, e.g., Nakamura et al., 1998; Narici et al., 1991; Penfield \& Rasmussen, 1950). If we consider this observation along with the fact that practice improves tactile sensitivity to stimuli presented to various body sites (see Craig \& Belser, 2006; Kóbor, Füredi, Kovács, Spence, \& Vidnyánszky, 2006), the question naturally arises as to whether there are differences in the duration and/or capacity of STM representations of stimuli presented on the fingers versus on the rest of the body surface. To investigate such possible differences, in Experiment 3 we compared the performance of participants in a partial-report task when tactile stimuli were presented on their fingertips versus across the rest of their body surface. Furthermore, since the fingers are easier to label verbally than the rest of the body surface, our final experiment also involved a concurrent articulatory suppression task.

\section{Experiment 1}

Experiment 1 had two aims: First, to investigate whether participants would demonstrate enhanced access to tactile STM for stimuli presented on their fingertips when performing a partial-report task, as compared to when they performed a numerosity judgment task. The second aim was to compare the effects of tactile and visual probes on participants' ability to report tactile stimuli during the partial-report task. In order to investigate these aims, the participants performed three experimental conditions. In the first condition, they had to report the total number of stimuli making up a display of up to six tactile stimuli presented on their fingertips (numerosity task). In the second condition, the participants had to detect whether or not the position indicated by a visual probe presented at a variable delay after the offset of a tactile display had previously contained a tactile stimulus (i.e., partial-report task with a visual probe). Finally, the participants had to 
determine whether or not a tactile stimulus had been presented at the position indicated by a tactile probe (i.e., partial-report task with a tactile probe).

\section{Method}

Participants A total of 16 participants (10 female, 6 male) took part in this experiment (mean age of 23 years, range from 19 to 26 years). All had normal or corrected-to-normal vision and reported normal tactile perception. They received a $£ 5$ (U.K.) gift voucher in return for their participation. The experiment took approximately $45 \mathrm{~min}$ to complete and was performed in accordance with the ethical standards laid down in the 1991 Declaration of Helsinki.

Materials and procedure The participants sat on a chair in an experimental chamber for the duration of the experiment. They were presented with vibrotactile stimuli delivered by means of eight resonant-type tactors (Part No. VBW32; Audiological Engineering Corp., Somerville, MA) with $1.6 \times 2.4 \mathrm{~cm}$ vibrating surfaces. The vibrators were driven by means of a custom-built nine-channel amplifier circuit (Haptic Interface Research Laboratory, Purdue University, Purdue, IN) that drove each tactor independently at $290 \mathrm{~Hz}$ (close to its resonant frequency). One tactor was placed on each finger of each of the participant's hands by means of Velcro strip belts. Green LEDs were mounted at the same position as each tactor but on the other side of the belts (see Fig. 1). The LEDs were driven by means of a custom-built relay box. The activation of each tactor and LED was controlled via the serial and parallel ports of a computer running custom software written in MATLAB 6.0. The intensity of each tactor was adjusted individually at the beginning of the experimental session in order that each vibrotactile stimulus could be perceived clearly and all of the vibrotactile stimuli were perceived to be of similar intensities. The amplification levels of the tactors were kept at their individually chosen levels throughout the experiment. White noise was presented over closed-ear headphones at $70 \mathrm{~dB}(\mathrm{~A})$ in order to mask any sound made by the activation of the tactors.

The participants completed three experimental conditions in three separate sessions. In one condition (the numerosity judgment task), the stimuli consisted of 200-ms-long vibrations delivered via a variable number of tactors. The number of tactors activated on each trial varied randomly between one and six. For each number of tactors, different patterns of activation were chosen randomly from among all possible stimulus combinations involving the participants' eight fingers (i.e., excluding their thumbs). The participants were instructed to indicate verbally the number of active tactors that they perceived on each trial. Their responses were recorded on the computer by the experimenter. Each number of tactors (i.e., from one to six) was presented 12 times, giving rise to a total of 72 trials for each participant.

In the second experimental condition (the partial-report task with a visual probe), a tactile display composed of one to six vibrotactile stimuli was presented to a participant's fingertips for $200 \mathrm{~ms}$. After a variable interval from the onset of the tactile display, one of the LEDs on the participant's body was illuminated for $50 \mathrm{~ms}$. Four intervals (i.e., stimulus onset asynchronies, or SOAs) between the tactile display and the onset of the light were used: 100 , $300,1,000$, and 2,500 ms. The participants placed their right foot on two foot pedals. They were instructed to raise their heel if the tactile stimulus had been presented in the position indicated by the light (i.e., hereafter "the visual
Fig. 1 Schematic figure highlighting the coincident and noncoincident probe location conditions in the partial-report task used in Experiment 1-3. On the left side, the black circles on the fingertips represent the active tactors, and the gray circles represent the inactive tactors. On the right side, the black circles represent the active LED (i.e., visual probe) or tactor (tactile probe)

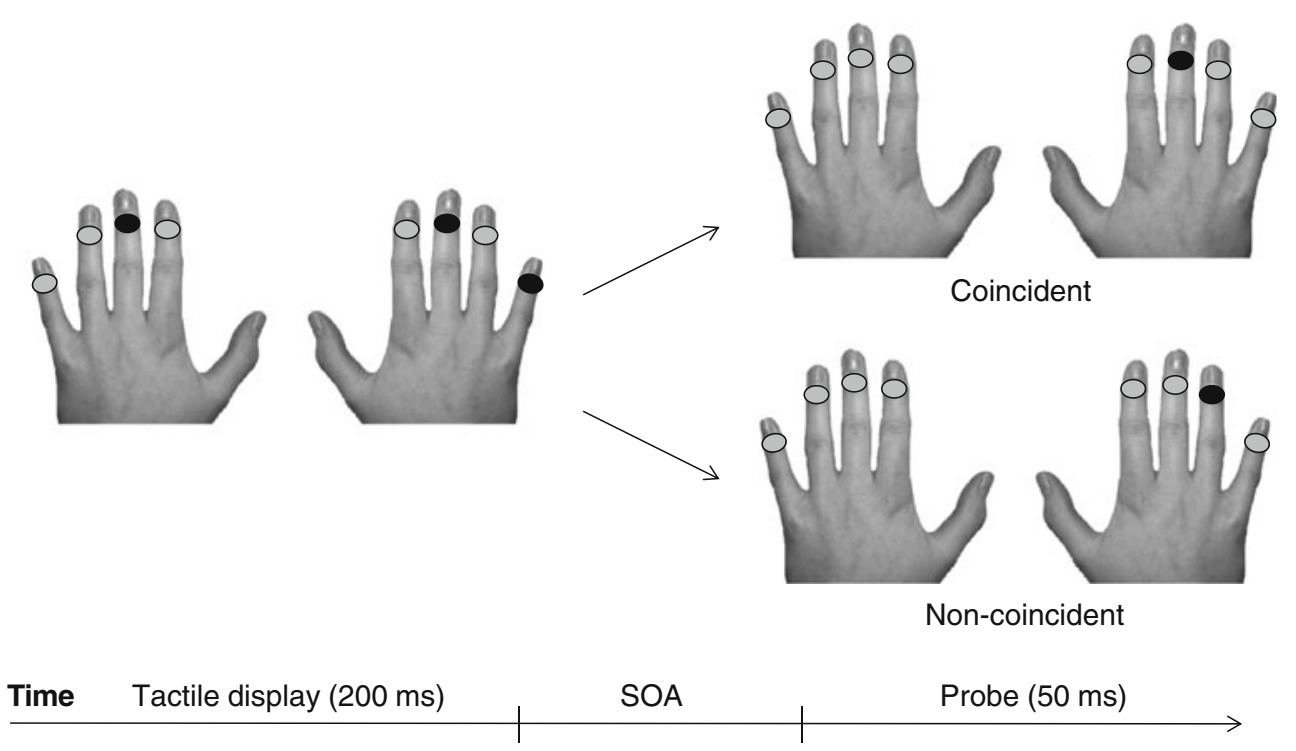


probe") and to raise their toe if no tactile stimulus had just been presented at the position indicated by the visual probe. Half of the participants performed the experiment with the reversed foot-pedal arrangement. The participants were instructed to respond as accurately as possible. In half of the trials, the probe was presented in a position that had been previously stimulated by one of the tactors (the coincident condition), while in the remaining trials the light was randomly presented in one of the other, unstimulated positions (the noncoincident condition). For each number of stimuli composing the display (one to six) and for each target-probe SOA, 10 trials were presented, giving rise to a total of 240 trials completed by each participant. The participants could make their unspeeded discrimination responses at any time up to $4,000 \mathrm{~ms}$ after the onset of the probe (at which point, the trial was terminated). No feedback was given regarding the correctness of a participant's responses.

The third experimental condition (the partial-report task with a tactile probe) was similar to the second condition, except that instead of a light, the probe consisted of the activation of one of the tactors for $50 \mathrm{~ms}$. The order of presentation of these three experimental conditions was randomly varied across participants. The participants were given 10 practice trials of the corresponding condition just before starting each of the three main experimental conditions.

\section{Results}

Trials on which the participants failed to make a response before the trial was terminated (less than $1 \%$ of trials overall) were not analyzed. The data concerning the mean percentages of errors in the numerosity judgment task were submitted to a repeated measures ANOVA with the factor of Number (six levels). This analysis resulted in a significant main effect $\left[F(5,75)=82.41, p<.0001, \eta_{\mathrm{p}}{ }^{2}=.85\right]$. A Duncan post-hoc test highlighted significant differences between each pair of consecutive numbers (all $p \mathrm{~s}<.05$ ), except for the comparison between five and six stimuli $(p=$ .9). For each number, the mean percentage of errors was then compared with chance-level performance $(83.3 \%$ errors; i.e., with six responses available to participants, the probability of responding correctly by chance equals $100 / 6=16.7 \%$ ) by means of planned $t$ tests. This analysis revealed that participants performed significantly better than chance for displays consisting of up to three stimuli (all $p \mathrm{~s}<.001)$, but not for displays composed of four $(p=.7)$, five, or six stimuli (note that performance for the displays composed of five and six stimuli was significantly different from chance, $p \mathrm{~s}<.05$, but in this case, the performance was significantly worse than chance level; see Fig. 2a). It should be noted that the errors typically consisted of participants underestimating the number of stimuli presented (see Fig. 2b). This result is consistent with previous studies using a similar task (see, e.g., Gallace, Tan, Haggard, \& Spence, 2008; Gallace, Tan, \& Spence, 2006, 2007).

The mean percentages of errors in the partial-report task were submitted to a repeated measures ANOVA with the factors of Modality (tactile vs. visual probe), Number (six levels), SOA (four levels), and Probe (coincident vs. noncoincident). The analysis revealed no significant effect of modality $\left[F(1,15)=3.13, p=.10, \eta_{\mathrm{p}}{ }^{2}=.11\right]$ or SOA $\left[F<1\right.$, n.s., $\left.\eta_{\mathrm{p}}{ }^{2}=.06\right]$, but it did reveal a significant main effect of number $\left[F(5,75)=59.20, p<.0001, \eta_{\mathrm{p}}{ }^{2}=.80\right]$. A Duncan post-hoc test performed on the Number factor revealed significant differences between each pair of consecutive numbers (all $p \mathrm{~s}<.01$ ), except for the comparison between five and six stimuli $(p=.13)$. Each time, the smaller the number, the better was participants' performance. The analysis also revealed a significant main effect of probe $\left[F(1,15)=6.91, p<.05, \eta_{\mathrm{p}}^{2}=.31\right]$, a significant interaction between number and probe $[F(5,75)=$ $\left.10.75, p<.0001, \eta_{\mathrm{p}}^{2}=.42\right]$, and a significant interaction between probe and SOA $\left[F(3,45)=14.54, p<.0001, \eta_{\mathrm{p}}{ }^{2}=\right.$ $.49]$. The analysis did not reveal any significant interaction between number and SOA $\left[F(15,225)=1.53, p=.10, \eta_{\mathrm{p}}{ }^{2}=\right.$ $.09]$, nor any other significant interaction. Note that the results of the partial-report task of Experiment 1, plotted as a function of the modality of the probe, are shown in Fig. $2 \mathrm{c}$ and d.

A Duncan post-hoc test conducted on the interaction between probe and number revealed that participants' performance was significantly worse when the probe location was coincident (i.e., when the probe was displayed on a finger that had previously been stimulated) than when it was presented on an unstimulated finger, for all displays (all $p \mathrm{~s}<.01$ ) except those consisting of just a single stimulus. A Duncan post-hoc test on the interaction between SOA and probe revealed that when the probe was coincident with one of the tactile stimuli, there were significant differences in performance between the $100-\mathrm{ms}$ SOA and all other SOAs (i.e., 300, 1,000, and 2,500 ms; all $p \mathrm{~s}<.05$ ), between the 300- and 2,500-ms SOAs, and between the 1,000 and 2,500 ms SOAs (all $p$ s $<.01$ ). In each case, the shorter the SOA between the tactile display and the probe, the better was participants' performance. With the presentation of a noncoincident probe, performance differed significantly between the $100-\mathrm{ms}$ SOA and all other SOAs (i.e., 300, 1,000, and 2,500 ms; all $p \mathrm{~s}<.05$ ), with worse performance being observed at the 100-ms SOA. Thus, participants' performance only decreased with increasing delays in the coincident probe condition.

For each modality, number, and SOA, the mean percentage of errors was compared with chance-level performance (i.e., $50 \%$ correct) using $t$ tests. This analysis revealed significant differences from chance for displays 


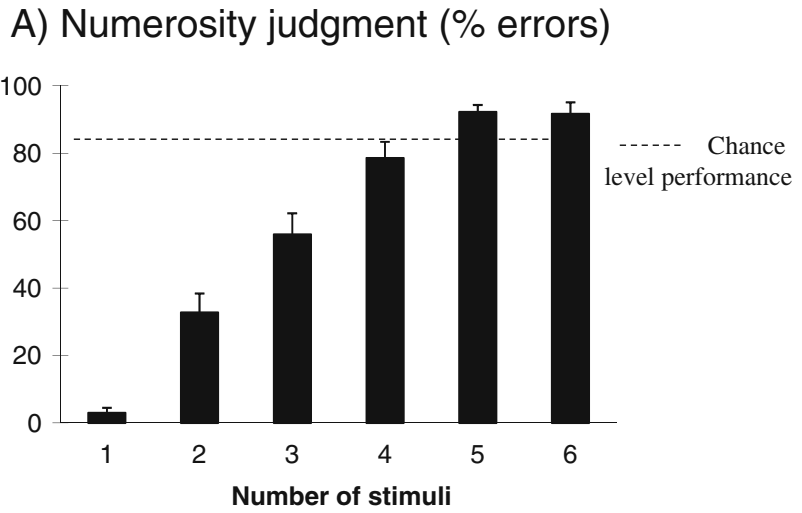

C) Partial report with tactile probe

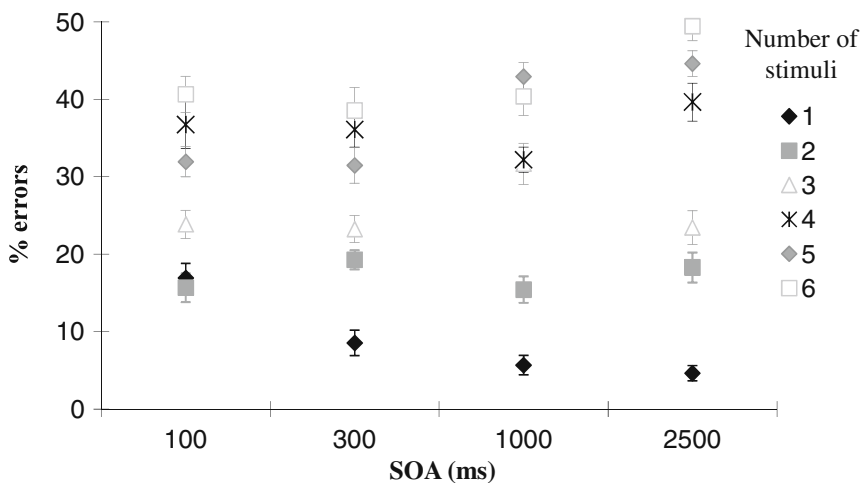

Fig. 2 Results of Experiment 1. (a) Mean error rates in the numerosity block of trials as a function of the number of tactors activated. Chance-level performance is represented by the dotted line. (b) Means of participants' responses in the numerosity block of trials

composed of one to four stimuli for all SOAs in both modality conditions (all $p \mathrm{~s}<.01$ ). For displays composed of five stimuli, performance was significantly different from chance for all SOAs (all $p \mathrm{~s}<.05$ ), except for the 1,000- and 2,500-ms SOAs in the tactile probe condition and the 300and 2,500-ms SOAs in the visual probe condition. For displays composed of six stimuli, performance was significantly different from chance for all combinations of stimulus presentation $(p s<.05)$, except at the 2,500-ms SOA in the tactile probe condition and the 100-, 1,000-, and 2,500-ms SOAs in the visual probe condition.

Given that the difference between the tactile and visual probe conditions did not result in any significant differences in our initial analysis, the data obtained from these two conditions were collapsed in our subsequent analysis. The mean percentages of errors in these two grouped conditions were subsequently compared with chance-level performance using $t$ tests. It should be noted here that when the two groups were collapsed (and only in this case), the total number of trials involved in the computation of chance-level performance for each of the different conditions was greater for the partial-report task than for the numerosity task. The
B) Numerosity judgment (number reported)

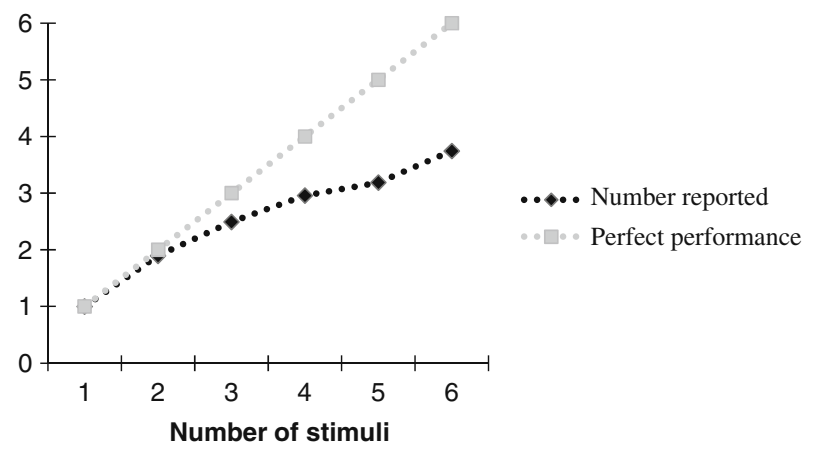

D) Partial report with visual probe

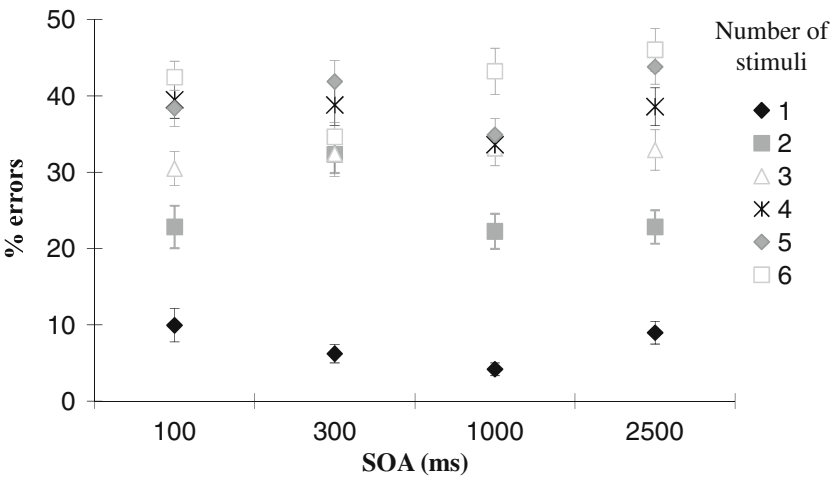

as a function of the number of tactors activated. (C\&D) Mean error rates as a function of the number of tactors activated and SOA for the partial-report block of trials with a tactile probe (c) and with a visual probe (d). Error bars represent the standard errors of the means

results revealed that participants' performance was significantly better than chance for all combinations of stimuli $(p \mathrm{~s}<.05)$ except for six stimuli at the 2,500-ms SOA.

\section{Discussion}

The first important result to emerge from the analysis of Experiment 1 was that participants were only able to identify at above chance-level performance up to three stimuli in the numerosity judgment task. However, when using a partial-report procedure, the participants were able to report at above chance-level performance whether or not a given finger has been stimulated when four fingers were stimulated, and this was true no matter what the SOA. They were also able to respond appropriately in the task when as many as six fingers were stimulated at the shortest SOAs. Thus, the identification of the stimulated fingers, although not accessible in terms of the full report of the total number of stimuli that had been presented, nevertheless remained accessible when participants performed a partial-report task with an appropriate poststimulus probe. This result is 
consistent with previous findings in the visual (e.g., Sperling, 1960) and auditory (Darwin et al., 1972) modalities, as well as with findings in the tactile modality when the stimuli were displayed across the body surface (Gallace, Tan, Haggard, \& Spence, 2008).

It should be noted here that the better performance in the partial-report task than in the numerosity judgment task cannot be accounted for simply in terms of potential differences in the difficulty of performing the second task. In order to investigate this hypothesis, Gallace, Tan, and Spence (2008) performed a control experiment using materials similar to those utilized in the present study. In that experiment, the participants performed both a task in which they had to report the number of stimuli that were presented (a numerosity judgment task) and a task in which they had to report the position of all of the stimuli that were presented (a position report task). The results revealed better performance in the numerosity judgment task than in the position report task. These results therefore indicate that the numerosity judgment task was not more difficult than the full position report task.

The second important result to emerge from the analysis of the results of Experiment 1 was that performance in the partial-report task was unaffected by the modality of the probe. Two hypotheses can be put forward to explain this particular result. First, it might be the case that the visual probe is translated into a tactile form for comparison with tactile memory. Alternatively, it might also be the case that information regarding the spatial positions that have been stimulated via a given sensory modality is not stored exclusively in a format specific to the modality of presentation of the stimuli. It has been suggested elsewhere that some spatial stimulus properties are extracted and held in a more abstract, possibly amodal, format (e.g., see Abravanel, 1981; see also Auvray, Gallace, Tan, \& Spence, 2007). The latter view can also be related to the literature on visual STM. In particular, it has previously been suggested that the information from a visual scene that is stored for later report or comparison is typically represented in an abstract, nonvisual code (see Irwin \& Andrew, 1996, for a review).

Finally, in Experiment 1, there was a difference in performance as a function of whether or not the probe was displayed at a previously stimulated location, a result that occurred in the partial-report task. First, participants' performance was better overall with noncoincident probes than with probes presented at a coincident location. In addition, the results revealed that, under conditions in which the probe location was coincident, the longer the interval between the presentation of the tactile display and the probe, the worse participants' performance became. Thus, in this experiment, the encoding of the positions that were tactually stimulated was subject to temporal decay. However, given that the question of the nature of this encoding remains open, Experiment 2 was designed to investigate whether or not the knowledge of the locations that were stimulated was stored semantically.

\section{Experiment 2}

Experiment 2 was designed to investigate the nature of the encoding of the positions of the tactually stimulated fingers. It might be argued that the locations of the stimuli on the participants' fingers could have favored a linguistic encoding of spatial location (see Kemmerer, 2006, for a review). In order to investigate whether or not knowledge concerning the stimulated locations is semantic in nature, Experiment 2 involved a concurrent articulatory suppression task. This verbal dual task involving interference was included in order to diminish the verbal resources that the participants would have available to them for encoding the tactually stimulated locations (see Mahrer \& Miles, 2002; Miles \& Borthwick, 1996). We hypothesized that if participants' ability to report the stimulated fingers was not due to a verbal encoding of the spatial location, the concurrent task should not have any effect other than to result in an overall decrease in their performance. In particular, there should not be any interaction between SOA and the presence versus absence of such a concurrent task. Indeed, it has been hypothesized that a global decrease in performance without any interaction with SOA suggests that the decrease in performance is due to the difficulty of performing a concurrent task, and should not be attributed entirely to the impossibility of using a verbal rehearsal strategy (see Mahrer \& Miles, 2002).

\section{Method}

A group of 20 new participants (12 female, 8 male; mean age of 23.5 years, range from 19 to 32 years) took part in this experiment. All of the participants reported normal tactile perception. The experiment took approximately $45 \mathrm{~min}$ to complete, and the participants received a $£ 5$ gift voucher in return for their participation.

The experimental setup and procedure were the same as for Experiment 1, with the following exceptions. In addition to the numerosity judgment block, participants completed two partial-report blocks with a tactile probe. One of these blocks was performed in the same manner as in Experiment 1, but in the second block, the participants were instructed to repeat the letters " $\mathrm{A}$ " and " $\mathrm{B}$ " continuously while performing the main detection task (i.e., a partial report with concurrent articulatory suppression task). As in Experiment 1, the SOAs between the presentation of the vibrotactile pattern and the presentation of the tactile 
probe were set at 100, 300, 1,000, and 2,500 ms. In each of the two partial-report blocks, 10 trials were presented for each number of stimuli presented and for each SOA, giving rise to a total of 480 trials completed by each participant for the two partial-report blocks.

\section{Results}

Trials in which the participants failed to give a response before the trial was terminated (less than $1 \%$ of trials overall) were not analyzed. The mean percentage of errors in the numerosity judgment block was submitted to a repeated measures ANOVA with the factor of Number (six levels). This analysis resulted in a significant effect $\left[F(5,95)=190.38, p<.0001, \eta_{\mathrm{p}}{ }^{2}=.91\right]$. A Duncan posthoc test highlighted significant differences between each pair of consecutive numbers (all $p \mathrm{~s}<.01$ ), except for the comparison between five and six stimuli $(p=.52)$. For each number, the mean percentage of errors was then compared with chance-level performance (i.e., $16.7 \%$ correct) using $t$ tests. This analysis revealed that performance was significantly better than chance for displays composed of up to three stimuli (all $p$ s $<.001$ ), but not for displays composed of four or more stimuli (performance for displays composed of five and six stimuli was significantly different from chance, $p \mathrm{~s}<.05$, but only because it was worse that chance level; see Fig. 3a).

The mean percentages of errors in the partial-report blocks were submitted to a repeated measures ANOVA with the factors of Filler Activity (silence vs. articulatory suppression), Number (six levels), SOA (four levels), and Probe (coincident vs. noncoincident). This analysis revealed significant main effects of filler activity $\left[F(1,19)=14.75, p<.01, \eta_{\mathrm{p}}^{2}=.40\right]$, number $\left[F(5,95)=96.11, p<.0001, \eta_{\mathrm{p}}^{2}=.83\right]$, SOA $[F(3,57)=$ $\left.6.05, p<.001, \eta_{\mathrm{p}}{ }^{2}=.24\right]$, and probe $[F(1,19)=26.05, p<$ $\left..001, \eta_{\mathrm{p}}{ }^{2}=.58\right]$, as well as a significant interaction between number and probe $\left[F(5,95)=23.04, p<.0001, \eta_{\mathrm{p}}{ }^{2}=.55\right]$. The analysis did not reveal any interactions between SOA and probe $\left[F(3,57)=1.15\right.$, n.s., $\left.\eta_{\mathrm{p}}{ }^{2}=.06\right]$, filler activity and SOA $\left[F(3,57)=1.21\right.$, n.s., $\left.\eta_{\mathrm{p}}^{2}=.06\right]$, filler activity and number $\left[F(5,95)<1\right.$, n.s., $\left.\eta_{\mathrm{p}}{ }^{2}=.02\right]$, or SOA and number $\left[F(15,285)<1\right.$, n.s., $\left.\eta_{\mathrm{p}}^{2}=.04\right]$, nor any other significant interaction.

A Duncan post-hoc test with the factor Number showed significant differences between each pair of consecutive numbers (all $p \mathrm{~s}<.01$ ), except for the comparison between the presentation of five and six stimuli $(p=.62)$. A Duncan post-hoc test conducted on the interaction between probe and number revealed that participants' performance was significantly worse when the probe location was coincident than when it was not for all display sizes (all $p \mathrm{~s}<.01$ ) excepting one or two stimuli.
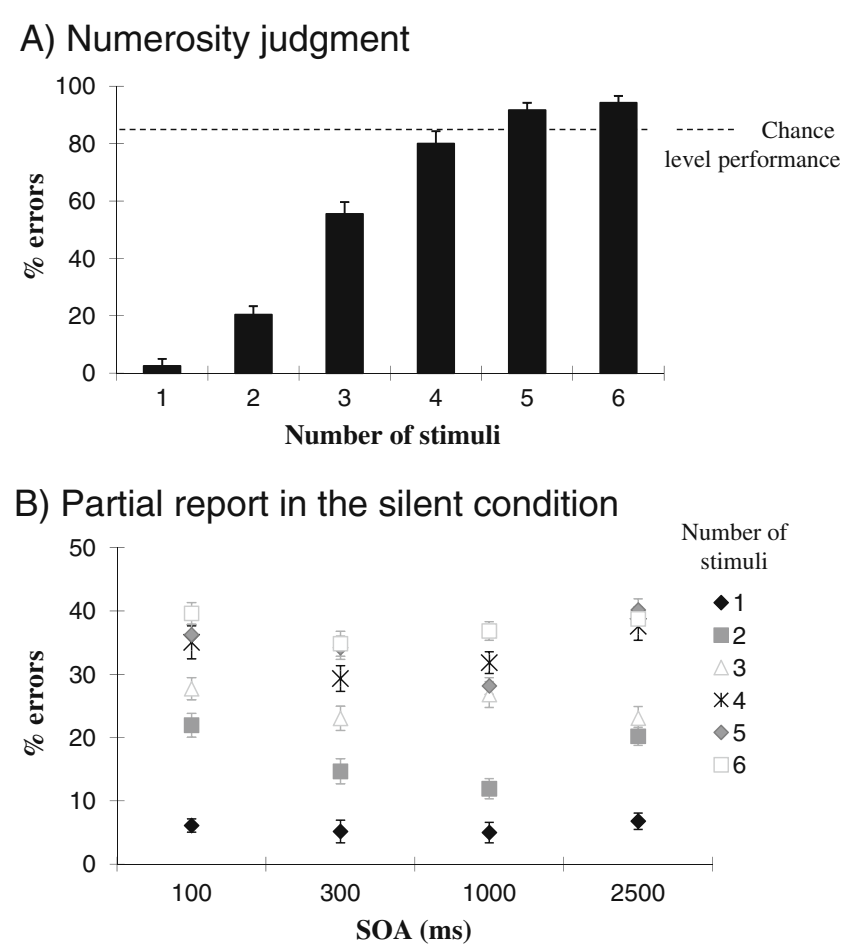

C) Partial report with verbal encoding control

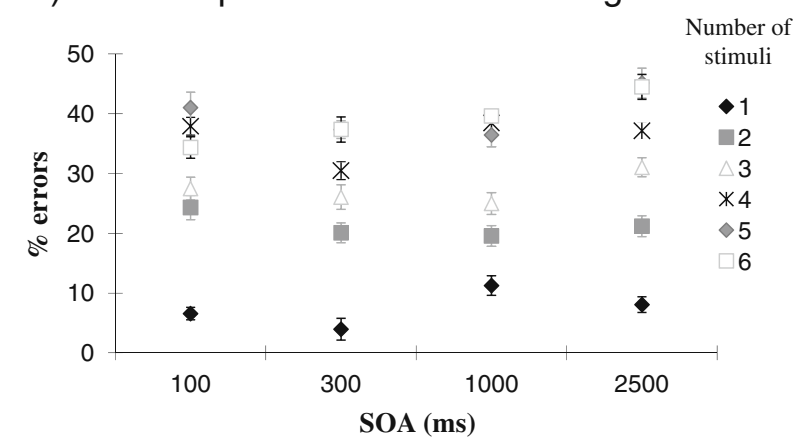

Fig. 3 Results of Experiment 2. (a) Mean error rates in the numerosity block of trials as a function of the number of tactors activated. Chancelevel performance is represented by the dotted line. (b\&c) Mean error rates as a function of the number of tactors activated and SOAs for the partialreport block of trials in the silent condition (b) and with verbal encoding controls (c). Error bars represent the standard errors of the means

For each combination of filler activities, SOA, and numerosity, the mean percentages of errors were then compared with chance-level performance (i.e., $50 \%$ correct) using $t$ tests. This analysis revealed significant differences from chance for all combinations (all $p \mathrm{~s}<.01$ ) except the articulatory suppression condition for five and six stimuli at the 2,500-ms SOA.

\section{Discussion}

Performance in the partial-report task remained higher than in the numerosity task, and this was true no matter whether 
the participants performed a concurrent articulatory suppression task or not. Thus, consistent with the results of Experiment 1, the participants in Experiment 2 were only able to identify three stimuli correctly at a level above chance performance in the numerosity judgment task. However, when the partial-report procedure was used instead, participants performed at an above-chance level whether or not a given finger has been stimulated when up to six fingers received stimuli at the shortest SOAs; this was the case for both of the conditions of filler activity (silence and articulatory suppression).

The second important result to emerge from the analysis of the results of the partial-report task performed in Experiment 2 was that articulatory suppression resulted in a significant decrease in participants' performance as compared with the silent condition. However, there was no significant interaction between filler activity (silence vs. articulatory suppression) and SOA. The absence of such an interaction suggests that it is unlikely that the participants relied solely on linguistic processes to perform the partialreport task. Indeed, as will be further detailed in the General Discussion, two different processes - not mutually exclusive - may be involved in memory for tactile locations. The first process, a sensory form of tactile memory, is thought to be unaffected by rehearsal processes, to be subject to temporal decay, and to be present mainly at short intervals. The second, which involves a linguistic component, is affected by rehearsal processes and can be present at long intervals (see Gilson \& Baddeley, 1969). As a consequence, if the memory for tactile locations was only coded linguistically, first, performance should have been at chance in cases in which there was a concurrent articulatory suppression task, and second, performance should not have been affected by increasing SOAs. Note that, in Experiment 2, participants' performance was above chance level in the articulatory suppression condition and decreased with increasing delays.

Alternatively, if two different processes (sensory and linguistic) are involved, the results are then likely to show an interaction between filler activity and SOA (see Gilson \& Baddeley, 1969; Mahrer \& Miles, 2002; see also Jonides, Lewis, Nee, Lustig, Berman, \& Moore, 2008, for a review). Indeed, the results should indicate that the SOA factor has less effect in those conditions without articulatory suppression (since the memory of the target locations can be rehearsed and maintained over long intervals) and more effect in conditions with articulatory suppression (since the linguistic component is thought to be impaired, and the sensory form of memory is subject to temporal decay). Thus, the absence of any interaction between filler activity and articulatory suppression reported in Experiment 2 suggests that it is unlikely that the participants relied solely on a verbal encoding of which fingers had been stimulated.
Rather, the overall decrease in performance in the articulatory suppression conditions of Experiment 2 can also be attributed to such a resource-consuming procedure, which might have impaired the participants' capacity to remember for subsequent report the stimuli that had been presented (e.g., see de Fockert, Rees, Frith, \& Lavie, 2001), and/or to the fact that performing such a task ends up adding irrelevant information into STM (see, e.g., Neath \& Nairne, 1995).

Finally, the results obtained in the partial-report task revealed that participants exhibited worse performance when the probe was displayed at a previously stimulated location than when it was not. It thus seems that the participants' knowledge that "there was no stimulation at that location" is less prone to error than their access to the location that has been stimulated previously. To the best of our knowledge, such a result has not been reported previously in the STM literature when the stimuli happened to be displayed simultaneously. However, some data exist in the case of successively presented stimuli. For example, in a study by Sternberg (1966), participants were presented with sequences of one to six digits. Next, a test stimulus was displayed, and the participants had to determine whether or not it was part of the sequence (item recognition task). The low error rate $(1.4 \%)$ did not allow for comparison between positive and negative responses. However, analyses of the reaction time data revealed that participants were faster at saying that a stimulus was there than at reporting that a stimulus was not there. The results also revealed that the mean reaction times increased linearly with the length of the sequence, and that this occurred for both positive and negative responses.

It should be noted that, in a variation of this paradigm (the context recall task; see Sternberg, 1967), participants had to locate the test digit in the sequence. In other words, they had to report the digit that was displayed just after the test digit. The reaction time results were similar to those in the item recognition task: The latencies increased linearly with list length. According to Sternberg (1966), the fact that in the two tasks (item recognition and context recall) reaction times increased linearly with both positive and negative responses indicates that the process is not "self terminating" (i.e., a positive response is initiated as soon as a match occurs). Rather, the process is "exhaustive"; that is, all of the items in the list are compared to the test item before an answer is made. It is also worth mentioning here that, on an item recognition task similar to Sternberg's (1967) paradigm, Murray (1986) found that errors increased following articulatory suppression, while mean reaction times did not. The author hypothesized that articulatory suppression affects registration in memory for some items, but does not affect the recognition time required for other items in the list. In addition, Clifton and Tash (1973) 
reported that the rates of memory search were similar for letters, one-syllable words, and three-syllable words, although the time to encode the memory probe was greater in the latter case. Clifton and Tash took their results to suggest that the representations of remembered items are not articulatory.

\section{Experiment 3}

Experiment 3 was designed to investigate whether or not tactile stimuli are stored in similar manners when they are presented on the fingertips versus across the body surface. Indeed, the difference in people's degrees of practice (or previous experience of being stimulated) on the different body sites might lead to greater tactile STM capacity when the stimuli are presented on the fingertips than when they are presented on the rest of the body surface. Experiment 3 used a partial-report procedure for stimuli presented on these two body sites in order to investigate any such differences. In addition, given that the fingers are easier to label than the rest of the body surface, Experiment 3 also involved a condition with a concurrent articulatory suppression task. Although the results of Experiment 2 tended to indicate that it is unlikely that the participants verbally rehearsed the bodily locations that had been stimulated, any difference in the influence of such an articulatory suppression task on the two body sites might provide additional insights into the differences in tactile STM, as well as into any differences in participants' strategies for stimuli presented on the body versus on the fingertips.

\section{Method}

A group of 14 new participants ( 9 female, 5 male) took part in this experiment (mean age of 24 years, range from 18 to 33 years). All of the participants reported normal tactile perception. The experiment took approximately $45 \mathrm{~min}$ to complete, and the participants received a $£ 5$ gift voucher in return for their participation.

The participants completed four blocks of experimental trials involving a partial-report procedure similar to the one presented for Experiments 1 and 2, with the following exceptions. In the first block of trials, participants were presented with a tactile display composed of up to five stimuli from eight possible locations on their fingers. The task here was the same as in Experiment 2. In the second block of trials, participants performed the same task while at the same time performing a concurrent articulatory suppression task (i.e., repeating the letters "A" and "B" continuously). In the third and fourth blocks of trials, participants performed the same conditions as in the first and second blocks, but this time the tactile stimuli were presented on their backs instead of their fingertips. A large belt with Velcro strips was used to make sure that the distances between the tactile stimuli were similar for the finger and bodily locations. At the beginning of the experiment, each participant placed his or her fingers on the belt in a comfortable position. The tactors were subsequently placed at these locations (under the participant's fingertips) on the belt and fixed by means of the Velcro strips. In the body condition, the tactors were kept at the same locations on the belt, which was placed on the participant's belly.

The SOAs between the presentation of the vibrotactile pattern and the probe were set at 1,000, 2,000, and $4,000 \mathrm{~ms}$. The presentation order of the four blocks of trials was randomly varied across participants. In each block, 8 trials were presented for each number and each SOA, giving rise to a total of 480 trials completed by each participant in the four blocks of trials. Before completing the experimental conditions, the participants were given 10 practice trials in each of the four conditions.

\section{Results}

Trials in which the participants failed to give a response before the trial was terminated (less than $4 \%$ of trials overall) were not analyzed. The mean percentages of errors were submitted to a repeated measures ANOVA with the factors of Location (finger vs. body), Filler Activity (silence vs. articulatory suppression), Number (five levels), SOA (three levels), and Probe (coincident vs. noncoincident). The analysis revealed a significant main effect of location $\left[F(1,13)=14.91, p<.01, \eta_{\mathrm{p}}{ }^{2}=.53\right]$, with better performance being reported when the stimuli were presented on the participants' fingertips than when they were presented across their body surface. The analysis also revealed a significant main effect of number $[F(4,52)=$ 94.75, $\left.p<.0001, \eta_{\mathrm{p}}{ }^{2}=.87\right]$, with performance decreasing with increasing display size (see Fig. 4). The analysis did not reveal any significant main effect of filler activity $\left[F(1,13)=3.02, p=.10, \eta_{\mathrm{p}}{ }^{2}=.18\right]$, probe $\left[F<1\right.$, n.s., $\eta_{\mathrm{p}}{ }^{2}=$ $.06]$, or SOA $\left[F<1\right.$, n.s., $\left.\eta_{\mathrm{p}}{ }^{2}=.07\right]$. However, the analysis did reveal a significant interaction between probe and number $\left[F(4,52)=9.11, p<.0001, \eta_{\mathrm{p}}{ }^{2}=.41\right]$, between filler activity and number $\left[F(4,52)=2.89, p<.05, \eta_{\mathrm{p}}{ }^{2}=\right.$ $.18]$, and between probe and SOA $[F(2,26)=5.04, p<.05$, $\left.\eta_{\mathrm{p}}{ }^{2}=.27\right]$. There were no other significant interactions.

A Duncan post-hoc test conducted on the interaction between probe and number revealed that performance was significantly worse when the probe location was coincident than when it was not for one, three, four, and five stimuli (all $p$ s $<.01$ ). A Duncan post-hoc test conducted on the 


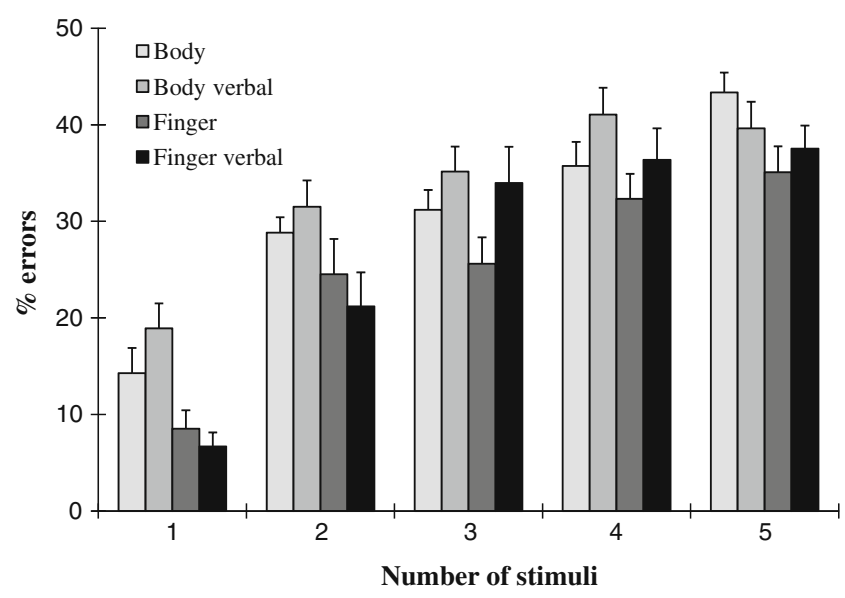

Fig. 4 Mean error rates as a function of the number of tactors activated for the two conditions of stimulus location (finger vs. body) and for the two filler activities (silence vs. verbal encoding control) in Experiment 3. Error bars represent the standard errors of the means

interaction between filler activity and number revealed that performance was significantly worse with filler activity for three and four stimuli $(p s<.05)$. Finally, a Duncan post-hoc test conducted on the interaction between probe and SOA revealed that when a coincident probe was presented, there were significant differences in performance between the 1,000 - and $4,000-\mathrm{ms}$ SOA conditions $(p<.01)$ and between the 2,000- and 4,000-ms SOA conditions $(p<.05)$, with worse performance being reported at the longer SOA. There were no significant differences between the different SOAs in the noncoincident probe condition.

\section{Discussion}

The first result to emerge from the analysis of the results of Experiment 3 was that participants' performance was better when the tactile pattern was presented to their fingertips than when it was displayed across the rest of their body surface. This was true no matter whether participants performed a concurrent articulatory suppression task or not. Such differences in tactile STM for the fingertips versus the body had been expected for at least two reasons. First, differences in the duration and/or capacity of shortterm representations of stimuli presented on the fingertips versus the rest of the body surface might arise from the fact that a relatively greater proportion of the somatosensory cortex is given over to the representation of the hands than to other parts of the body (e.g., Nakamura et al., 1998; Narici et al., 1991; Penfield \& Boldrey, 1937). That is, the size of the neural representations on the somatosensory cortex might differentially constrain the duration, capacity, and/or access to tactile information presented in different locations on the body surface. Indeed, it should be noted that the suggestion has been made that tactile short-term memories are organized somatotopically, following the organization of somatosensory cortex in the brain (e.g., Gallace \& Spence, 2009; Harris, Harris, \& Diamond, 2001). Second, most people are simply more experienced at discriminating tactile stimuli using their hands than using other parts of their body surface, and practice can lead to greater tactile sensitivity (e.g., see Hodzic, Veit, Karim, Erb, \& Godde, 2004, for an fMRI study highlighting the neural correlates of practice in tactile discrimination tasks).

The second important result to emerge from Experiment 3 was that participants' performance was impaired when they performed a concurrent articulatory suppression task (however, only when three and four stimuli were displayed). More importantly, there was no interaction between filler activity (silence vs. articulatory suppression) and SOA. Thus, it is unlikely that the participants verbally encoded the stimulated locations in any of the body site conditions. In addition, it should also be noted that the duration of stimulus presentation used in our experiments (200 ms) might have been short enough to have prevented participants from coding the bodily locations verbally. Relevant to this argument are the results of a study by Le Clec'H et al. (2000) in which participants were presented with written or spoken names of body parts. The average reaction time to determine whether or not a particular body part was higher than the shoulder of a standing person was greater than $860 \mathrm{~ms}$. In our study, the duration of tactile stimulus presentation was only $200 \mathrm{~ms}$, and the shortest SOA between stimulus presentation and the probe (which gave rise to the best performance) was set at $100 \mathrm{~ms}$. Thus, on the basis of Le Clec'H et al.'s data, it would appear unlikely that participants in the experiments reported here used a verbal code to perform the task.

\section{General discussion}

In the study reported here, we investigated tactile STM using both a numerosity task and a partial-report procedure. In Experiment 1, the participants were presented with displays consisting of up to six tactile stimuli presented on their fingertips. They had to either report the total number of stimuli composing the display or detect whether or not the position indicated by a probe (visual or tactile) had previously contained a tactile stimulus (partial-report task). The first important result to emerge from Experiment 1 was that participants were only able to identify three stimuli correctly in the numerosity judgment task. However, when using a partial-report procedure, they correctly reported up to six stimuli at the shortest SOAs. This result reveals that some of the tactile information regarding the identification of the fingers that were stimulated, although not available 
for full report in the numerosity task, could nevertheless still be accessed by means of a partial-report procedure.

It should be noted that previous visual studies have demonstrated that, at short intervals, the superiority of partial- over whole-report procedures is dependent on the selection criterion that is required to report the stimuli. Indeed, such a partial-report superiority effect has been found when selection was performed according to spatial location (e.g., report the items located at a specific position on a display, such as the top row; see Averbach \& Coriell, 1961; Sperling, 1960), color (e.g., report only the letters written in a specific color; see Clark, 1969; von Wright, 1968), brightness and size (von Wright, 1968), and shape (Turvey \& Kravetz, 1970). However, such a superiority effect was not found when participants had to report either the letters or the digits in a display (Sperling, 1960). Thus, the superiority of partial over whole report when the selection criterion was based on spatial location was extended in our study so as to include stimuli presented in the tactile modality.

The second result to emerge from the partial-report conditions of Experiment 1 was that worse performance was observed when the probe was displayed at a previously stimulated location than when it was displayed at another location. It thus appears that access to the stimulated locations is more prone to errors than is access to the unstimulated locations. Furthermore, performance declined with increasing delays specifically in those conditions in which the probe was displayed at a previously stimulated location. Note that similar results were reported for Experiment 3. One possible explanation for this result is that the process of responding is asymmetric for the positive set (i.e., the stimulated locations) and the negative set (i.e., the unstimulated locations). It may be that only the positive set is searched, and thus that only the memory of this set fades with increasing SOA. Alternatively, these results might suggest the presence of two processes. The first would be dependent on delays and would reflect the decay of a short-term tactile representation, similar to previous findings in vision (Sperling, 1960, 1963). The second process would be independent of delay and related to the fusion (or merging) of the tactually stimulated locations into an overall pattern. Indeed, although in Sperling's (1960) visual studies the letters had both a clear localization and a clear identity, the tactile stimuli used in our experiment only differed in their spatial component, which could somehow be fused in a compound pattern (or percept) whose elements could not be individuated easily. The probe might subsequently have allowed for the individuation and retrieval of those elements. In addition, it should be mentioned that since our tactile stimuli were presented simultaneously, this could have led to masking effects and contributed to participants' confusion at the level of the stimulus encoding (Alluisi, Morgan, \& Hawkes, 1965; see also Coltheart, 1980, for a similar impairment in visual and auditory STM resulting from the presence of masking). Such masking effects might have led to some asymmetries and/or inconsistencies in the results reported here.

The third important result to emerge from Experiment 1 was that in the partial-report task, participants' performance was similar, no matter whether the probe was visual or tactile. This result might be taken to suggest that the information regarding the fingers that were tactually stimulated was not stored in a strictly tactile format. This result is consistent with numerous previous studies that have examined the interaction between vision, touch, and proprioception and have revealed the existence of multisensory/amodal spatial representations that code external events with respect to an observer's body (see Auvray, Gallace, Hartcher-O'Brien, Tan, \& Spence, 2008; Gallace \& Spence, 2008; Spence \& Driver, 2004). In addition, this result might also be taken to suggest that conscious information processing in the tactile modality cannot be completely separated from the more general processing of spatial information (see Gallace \& Spence, 2008, for a review). In particular, tactile information regarding the locations that are stimulated sometimes involves a spatial component, in order to allow for knowledge of which part of the body was stimulated.

However, following on from the suggestion that the spatial information accessed through a given sensory modality is extracted and held in an amodal format (see Abravanel, 1981), the question arises as to whether or not the encoding of such spatial information is linguistic. To date, previous studies of tactile memory have investigated the influence of a concurrent articulatory suppression task on participants' performance with only a single stimulus or with several stimuli presented successively, but never when several tactile stimuli were presented simultaneously. Experiment 2 was designed to investigate the role of rehearsal processes in tactile STM for stimuli that had been presented simultaneously. To address this question, a partial-report task was used together with a concurrent articulatory suppression task. The main result to emerge from this experiment was that, although there was a global decrease in performance in the articulatory suppression condition as compared with the silent condition, there was no significant interaction with SOA. This means that it is unlikely that the participants performed the task solely by relying on linguistic processes.

It should be mentioned that, to date, no agreement has been reached concerning the nature of the encoding of tactile information. In the extant literature, most of the studies using a single tactile stimulus have adopted a tactile analogue of the Brown-Peterson distractor task (Brown, 
1958; Peterson \& Peterson, 1959). In this paradigm, an unseen tactile stimulus is presented on the participant's forearm. After a variable delay, the participant has to point at the previously stimulated location. Any decrease in the accuracy of participants' pointing with increasing delay is taken to suggest a decay of the tactile information stored in memory. During the delay period, the participants could either remain silent or perform a concurrent articulatory suppression task in order to interfere with any verbal rehearsal strategy that they might have been using. Using this procedure with SOAs varying from 0 to $60 \mathrm{~s}$, Gilson and Baddeley (1969) reported an interaction between SOA and filler activity (silence vs. articulatory suppression). In particular, in the articulatory suppression condition, the decline in accuracy was linear and reached asymptote after $45 \mathrm{~s}$. On the other hand, in the silent condition, accuracy was maintained with delays of up to $15 \mathrm{~s}$ and then declined, with asymptotic performance being reached by $60 \mathrm{~s}$.

Gilson and Baddeley (1969) interpreted their data as suggesting the existence of two tactile memory processes: A sensory form of tactile memory that is unaffected by articulatory suppression and is present mainly at short intervals, and another one observed at longer intervals, which is maintained by some rehearsal processes. However, using the same type of task, Sullivan and Turvey (1972) found no interaction between filler activity and SOA. Both in the silent and in the articulatory suppression conditions, the decline in performance reached asymptote after an interval of $5 \mathrm{~s}$. Thus, contrary to the results of Gilson and Baddeley's experiment, the absence of any interaction in the study by Sullivan and Turvey suggests a more unitary model of tactile memory, composed of a decaying and unrehearsable form of storage.

In addition, Miles and Borthwick (1996) used the same tactile Brown-Peterson paradigm with three filler activity conditions: silence, articulatory suppression, and tactile interference. Performance was impaired in both the articulatory suppression and tactile interference conditions, as compared with the silent condition. In addition, no interaction was found between filler activity and SOA. The authors concluded that articulatory suppression and tactile interference operate on different mechanisms underlying the ability to recall tactile stimuli. That is, tactile interference reduces the discriminability of the target location, whereas articulatory suppression diminishes the central processing resources involved in the memorization of the original target location. Miles and Borthwick underlined the fact that such memory processes need not necessarily be underpinned by an articulatory code. Overall, these studies, using a single stimulus presentation, support the existence of a tactile STM system. However, no convergence was found concerning either the nature of the encoding of tactile information or the precise rate of temporal decay.
Mahrer and Miles (2002) investigated people's memory for sequences of tactile stimuli. In their study (Exp.1), participants had to assess the similarity of two tactile sequences. The two sequences were presented at variable delays with three conditions of filler activity: silence, articulatory suppression, and tactile interference. The results revealed that performance was impaired by between-sequence articulatory suppression, but not by either silence or tactile interference. This result suggests that the encoding of tactile sequences has a verbal component (and therefore can be rehearsed). However, the participants' performance remained higher than chance even in the articulatory suppression condition. The authors thus suggested that additional encoding strategies may have been involved, and in particular, they suggested that participants use spatial imagery (for the link between touch and imagery, see also Heller, 1980; Warren \& Rossano, 1991), which has been shown to be immune to concurrent articulatory suppression (see, e.g., Logie, 1986). To investigate this proposal, the authors used a diagrammatic representation of the hand (Mahrer \& Miles, 2002, Exp.3) and showed that seeing the second sequence presented either to the hand or on a diagrammatic representation of a hand improved recognition, as compared with a condition in which the second sequence was only presented in the tactile modality. The results of Mahrer and Miles's study therefore reveal that our memory for tactile sequences does not rely solely on the retention of sensory traces, but also involves the deployment of strategies utilizing a combination of verbal rehearsal and visuospatial recoding.

In the present study, we found (in Exp.2) a global decrease in performance in the articulatory suppression condition as compared with the silent condition, with no significant interaction with SOA. This result could be taken to suggest that, in the paradigm used here, it is unlikely that participants relied solely on a verbal code to perform the task. It might be the case that, in these experiments, the simultaneous presentation of stimuli as well as the use of very short delays impaired the participants' ability to rely on verbal encoding of the locations where the tactile stimuli were presented. In addition, the results of Experiment 2 also revealed that participants' performance remained higher in the partial-report than in the numerosity task, and that this was the case no matter whether the participants performed a concurrent articulatory suppression task or not.

Finally, Experiment 3 was designed to investigate whether or not simultaneously presented tactile stimuli are encoded similarly when they are presented on the fingertips versus when they are presented across the rest of the body surface. We hypothesized that the greater sensitivity and practice of experiencing/discriminating tactile stimuli on the fingertips than on the rest of the body (see Weber, 1846, and Weinstein, 1968, for pioneering work on variations in 
tactile sensitivity between different body sites) might differentially constrain the duration, capacity, and/or access to tactile information presented in different locations on the body surface. Consistent with this view, a study by Murray, Ward, and Hockley (1975) successfully highlighted the link between tactile sensitivity and tactile memory. They determined the "tactile sensitivity" of 12 different body sites by measuring the smallest distance between two stimulated locations that were indistinguishable from the stimulation of just a single point (two-point discrimination threshold). The researchers then determined a "memory threshold" for six of these body sites by touching two nearby points at these locations with variable delays between them and asking the participants whether or not they thought they had been touched in the same place on both occasions. The authors found that the higher the tactile sensitivity of a given body location, the lower the degree, or rate, of forgetting at that location. The results of Experiment 3 in the present study revealed better performance when the stimuli were presented to the fingertips than to the rest of the body surface. However, the results did not reveal any other differences between the two locations of stimulus presentation. Thus, greater sensitivity on the fingertips than on the rest of the body surface would seem to involve greater STM capacity but no difference in encoding processes.

In summary, the experiments reported here show, with procedures similar to those used previously in visual studies, that short-lasting information regarding tactile stimuli that is not accessible during full-report procedures can still be accessed when partial-report procedures are used instead. In addition, the three experiments reported here allow further clarification concerning the nature of such tactile STM. In particular, they reveal that information concerning the locations that were tactually stimulated is not stored in an exclusively tactile format (Exp.1). In addition, such encoding of spatial locations is not solely linguistic in nature (Exps. 2 and 3). Finally, the STM capacity for stimuli presented on the fingertips is greater than for stimuli presented across the rest of the body surface, in the absence of any difference in the encoding processes (Exp.3).

\section{References}

Abravanel, E. (1981). Integrating the information from eyes and hands: A developmental account. In R. D. Walk \& H. L. Pick Jr. (Eds.), Intersensory perception and sensory integration (pp. 71-108). New York: Plenum Press.

Alluisi, E. A., Morgan, B. B., Jr., \& Hawkes, G. R. (1965). Masking of cutaneous sensations in multiple stimulus presentations. Perceptual and Motor Skills, 20, 39-45.

Atkinson, J., Campbell, F. W., \& Francis, M. R. (1976). The magic number $4 \pm 0$ : A new look at visual numerosity judgements. Perception, 5, 327-334.
Auvray, M., Gallace, A., Hartcher-O’Brien, J., Tan, H. Z., \& Spence, C. (2008). Tactile and visual distractors induce change blindness for tactile stimuli presented on the fingertips. Brain Research, $1213,111-119$.

Auvray, M., Gallace, A., Tan, H. Z., \& Spence, C. (2007). Crossmodal change blindness between vision and touch. Acta Psychologica, 126, 79-97.

Averbach, E., \& Coriell, A. S. (1961). Short-term memory in vision. Bell System Technical Journal, 40, 309-328.

Bliss, J. C., Crane, H. D., Mansfield, P. K., \& Townsend, J. T. (1966). Information available in brief tactile presentations. Perception \& Psychophysics, 1, 273-283.

Brown, J. (1958). Some tests of the decay theory of immediate memory. The Quarterly Journal of Experimental Psychology, 10, $12-21$.

Camos, V., \& Tillmann, B. (2008). Discontinuity in the enumeration of sequentially presented auditory and visual stimuli. Cognition, 107, 1135-1143.

Chi, M. T., \& Klahr, D. (1975). Span and rate of apprehension in children and adults. Journal of Experimental Child Psychology, 19, 434-439.

Clark, S. E. (1969). Retrieval of color information from the preperceptual storage system. Journal of Experimental Psychology, 82, 263-266.

Clifton, C., Jr., \& Tash, J. (1973). Effect of syllabic word length on memory-search rate. Journal of Experimental Psychology, 99, 231-235.

Coltheart, M. (1980). Iconic memory and visible persistence. Perception \& Psychophysics, 27, 183-228.

Coltheart, M. (1983). Iconic memory. Philosophical Transactions of the Royal Society B, 302, 283-294.

Cowan, N. (1984). On short and long auditory stores. Psychological Bulletin, 96, 341-370.

Cowan, N. (2001). The magical number 4 in short-term memory: A reconsideration of mental storage capacity. Behavioral and Brain Sciences, 24, 87-185.

Craig, J. C., \& Belser, A. N. (2006). The crossed-hands deficit in tactile temporal-order judgments: The effect of training. Perception, $35,1561-1572$.

Darwin, C., Turvey, M. T., \& Crowder, R. G. (1972). An analogue of the Sperling partial report procedure: Evidence for brief auditory storage. Cognitive Psychology, 3, 255-267.

de Fockert, J. W., Rees, G., Frith, C. D., \& Lavie, N. (2001). The role of working memory in visual selective attention. Science, 291, 1803-1806.

Fernberger, S. W. (1921). A preliminary study of the range of visual apprehension. The American Journal of Psychology, 32, 133.

Gallace, A., \& Spence, C. (2008). The cognitive and neural correlates of "tactile consciousness": A multisensory perspective. Consciousness and Cognition, 17, 370-407.

Gallace, A., \& Spence, C. (2009). The cognitive limitations and neural correlates of tactile memory. Psychological Bulletin, 135, 380406.

Gallace, A., Tan, H. Z., Haggard, P., \& Spence, C. (2008). Short term memory for tactile stimuli. Brain Research, 1190, 132-142.

Gallace, A., Tan, H. Z., \& Spence, C. (2006). Numerosity judgments for tactile stimuli distributed over the body surface. Perception, $35,247-266$.

Gallace, A., Tan, H. Z., \& Spence, C. (2007). Multisensory numerosity judgments for visual and tactile stimuli. Perception \& Psychophysics, 69, 487-501.

Gallace, A., Tan, H. Z., \& Spence, C. (2008). Can tactile stimuli be subitized? An unresolved controversy within the literature on numerosity judgments. Perception, 37, 782-800.

Gilson, E. Q., \& Baddeley, A. D. (1969). Tactile short-term memory. The Quarterly Journal of Experimental Psychology, 21, 180-184. 
Hancock, P. A., Oron-Gilad, T., \& Szalma, J. L. (2007). Elaborations of the multiple-resource theory of attention. In A. F. Kramer, D. A. Wiegmann, \& A. Kirlik (Eds.), Attention: From theory to practice (pp. 45-56). Oxford: Oxford University Press.

Harris, J. A., Harris, I. M., \& Diamond, M. E. (2001). The topography of tactile working memory. The Journal of Neuroscience, 21, $8262-8269$.

Heller, M. A. (1980). Tactile retention: Reading with the skin. Perception \& Psychophysics, 27, 125-130.

Hill, J. W., \& Bliss, J. C. (1968). Modelling a tactile sensory register. Perception \& Psychophysics, 4, 91-101.

Hodzic, A., Veit, R., Karim, A. A., Erb, M., \& Godde, B. (2004). Improvement and decline in tactile discrimination behaviour after cortical plasticity induced by passive tactile coactivation. The Journal of Neuroscience, 24, 442-446.

Irwin, D. E., \& Andrew, R. V. (1996). Integration and accumulation of information across saccadic eye movements. In T. Inui \& J. L. McClelland (Eds.), Attention and performance XVI: Information integration in perception and communication (pp. 125-155). Cambridge, MA: MIT Press.

James, W. (1950). The principles of psychology. New York: Dover. (Original work published 1890)

Jevons, W. G. (1871). The power of numerical discrimination. Nature, $3,281-282$.

Jonides, J., Lewis, R. L., Nee, D. E., Lustig, C. A., Berman, M. G., \& Moore, K. S. (2008). The mind and brain of short-term memory. Annual Review of Psychology, 59, 193-224.

Kashino, M., \& Hirahara, T. (1996). One, two, many-Judging the number of concurrent talkers. The Journal of the Acoustical Society of America, 99, 2596-2603.

Kaufman, E., Lord, M., Reese, T., \& Volkmann, J. (1949). The discrimination of visual number. The American Journal of Psychology, 62, 498-525.

Kemmerer, D. (2006). The semantics of space: Integrating linguistic typology and cognitive neuroscience. Neuropsychologia, 44, 1607-1621.

Kóbor, I., Füredi, L., Kovács, G., Spence, C., \& Vidnyánszky, Z. (2006). Back-to-front: Improved tactile discrimination performance in the space you can't see. Neuroscience Letters, 400, 163-167.

Le Clec'H, G., Dehaene, S., Cohen, L., Mehler, J., Dupoux, E., Poline, J. B., et al. (2000). Distinct cortical areas for names of numbers and body parts independent of language and input modality. Neuroimage, 12, 381-391.

Logie, R. H. (1986). Visuo-spatial processes in working memory. The Quarterly Journal of Experimental Psychology, 38, 229-247.

Mahrer, P., \& Miles, C. (2002). Recognition memory for tactile sequences. Memory, 10, 7-20.

Mandler, G., \& Shebo, J. B. (1982). Subitizing: An analysis of its component processes. Journal of Experimental Psychology: General, 111, 1-22.

Miles, C., \& Borthwick, M. (1996). Tactile short-term memory revisited. Memory, 4, 655-668.

Murray, D. J. (1986). Level of articulation and short-term recognition following brief probe delays. Bulletin of the Psychonomic Society, 24, 103-106.

Murray, D. J., Ward, R., \& Hockley, W. E. (1975). Tactile short-term memory in relation to the two point threshold. The Quarterly Journal of Experimental Psychology, 27, 303-312.

Nakamura, A., Yamada, T., Goto, A., Kato, T., Ito, K., Abe, Y., et al. (1998). Somatosensory homunculus as drawn by MEG. Neuroimage, 74, 377-386.
Narici, L., Modena, I., Opsomer, R. J., Pizzella, V., Romani, G. L., Torrioli, G., et al. (1991). Neuromagnetic somatosensory homunculus: A noninvasive approach in humans. Neuroscience Letters, 121, 51-54.

Neath, I., \& Nairne, J. S. (1995). Word-length effects in immediate memory: Overwriting trace-decay theory. Psychonomic Bulletin \& Review, 2, 429-441.

Neisser, U. (1967). Cognitive psychology. New York: AppletonCentury-Crofts.

Penfield, W., \& Boldrey, E. (1937). Somatic motor and sensory representation in the cerebral cortex of man as studied by electrical stimulation. Brain, 60, 389-443.

Penfield, P., \& Rasmussen, T. (1950). The cerebral cortex of man: A clinical study of localization of function. New York: Macmillan.

Peterson, L. R., \& Peterson, M. J. (1959). Short-term retention of individual verbal items. Journal of Experimental Psychology, 58, 193-198.

Peterson, S., \& Simon, T. J. (2000). Computational evidence for the subitizing phenomenon as an emergent property of the human cognitive architecture. Cognitive Science, 24, 93-122.

Riggs, K. J., Ferrand, L., Lancelin, D., Fryziel, L., Dumur, G., \& Simpson, A. (2006). Subitizing in tactile perception. Psychological Science, 17, 271-275.

Simons, D., \& Langheinrich, D. (1982). What is magic about the magical number four? Psychological Research, 44, 283-294.

Spence, C., \& Driver, J. (Eds.). (2004). Crossmodal space and crossmodal attention. Oxford: Oxford University Press.

Sperling, G. (1960). The information available in brief visual presentations. Psychological Monographs, 74, 1-29.

Sperling, G. (1963). A model for visual memory tasks. Human Factors, 5, 19-31.

Sternberg, S. (1966). High-speed scanning in human memory. Science, 153, 652-654.

Sternberg, S. (1967). Retrieval of contextual information from memory. Psychonomic Science, 8, 55-56.

Sullivan, E. V., \& Turvey, M. T. (1972). Short-term retention of tactile stimulation. The Quarterly Journal of Experimental Psychology, 24, 253-261.

Treisman, M., \& Roston, A. B. (1972). Brief auditory storage: A modification of Sperling's paradigm applied to audition. Acta Psychologica, 36, 161-170.

Trick, L. M., \& Pylyshyn, Z. W. (1994). Why are small and large number enumerated differently? A limited-capacity preattentive stage in vision. Psychological Review, 101, 80-102.

Turvey, M. T., \& Kravetz, S. (1970). Retrieval from iconic memory with shape as the selection criterion. Perception \& Psychophysics, $8,171-172$.

von Wright, J. M. (1968). Selection in visual immediate memory. The Quarterly Journal of Experimental Psychology, 20, 62-68.

Warren, D. H., \& Rossano, M. J. (1991). Inter modality relations: Vision and touch. In M. A. Heller \& W. Schiff (Eds.), The psychology of touch (pp. 119-137). Hillsdale, NJ: Erlbaum.

Weber, E. H. (1846). Der Tastsinn und das Gemeingefühl [The sense of touch and the common sensibility]. In R. Wagner (Ed.), Handwörterbuch der Physiologie (pp. 481-588). Braunschweig: Vieweg.

Weinstein, S. (1968). Intensive and extensive aspects of tactile sensitivity as a function of body part, sex, and laterality. In D. R. Kenshalo (Ed.), The skin senses (pp. 195-222). Springfield, IL: Charles C. Thomas.

Wickens, C. D. (1984). Processing resources in attention. In R. Parasuraman \& D. R. Davies (Eds.), Varieties of attention (pp. 63-102). Orlando: Academic Press. 